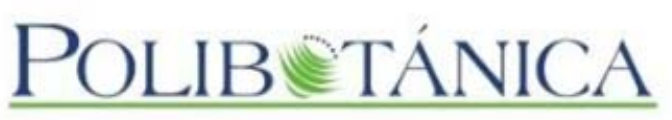

\title{
Polibotánica
}

ISSN electrónico: 2395-9525

polibotanica@gmail.com

Instituto Politécnico Nacional

México

http:www.polibotanica.mx

\section{VIABILIDAD Y GERMINACIÓN DE SEMILLA DE Cordia elaeagnoides A. DC.}

\section{SEED VIABILITY AND GERMINATION OF Cordia elaeagnoides A. DC.}

Solís-Sandoval, S.; M. Gómez-Romero, y C. Velázquez-Becerra.

VIABILIDAD Y GERMINACIÓN DE SEMILLA DE Cordia elaeagnoides A. DC. SEED VIABILITY AND GERMINATION OF Cordia elaeagnoides A. DC.

POLIBETANICA Instituto Politécnico Nacional
Núm. 48: 121-134 México. Julio 2019

DOI: $10.18387 /$ polibotanica. 48.10

(C) (i) (2) Este es un artículo de acceso abierto bajo la licencia Creative Commons 4.0 Atribución-No Comercial (CC BY-NC 4.0 Internacional). 
VIABILIDAD Y GERMINACIÓN DE SEMILLA DE Cordia elaeagnoides A. DC.

\section{SEED VIABILITY AND GERMINATION OF Cordia elaeagnoides A. DC.}

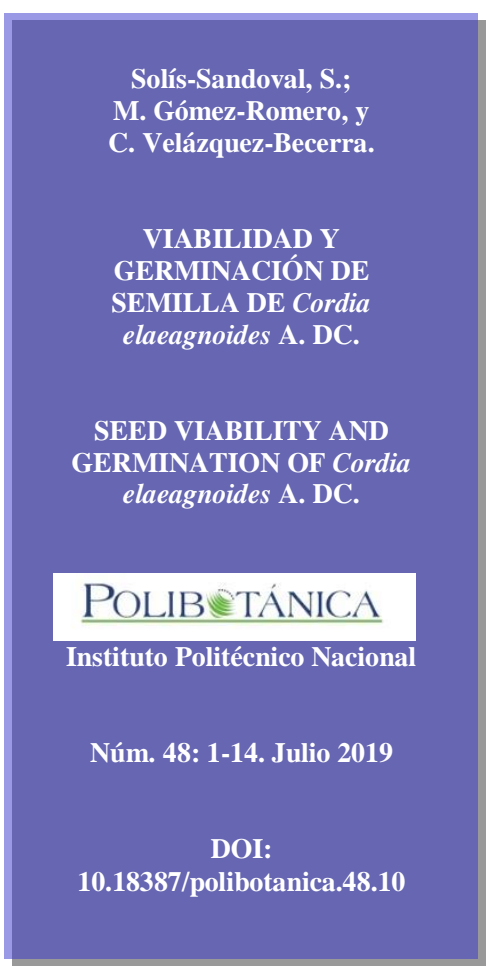

\begin{abstract}
S. Solís-Sandoval
Facultad de Ingeniería en Tecnología de la Madera, Laboratorio de Microbiología de la Madera, Universidad Michoacana de San Nicolás de Hidalgo. Edificio D, Ciudad Universitaria. CP 58030 Morelia, Michoacán, México.
\end{abstract}

M. Gómez-Romero
Instituto de Investigaciones Químico Biológicas,
Universidad Michoacana de San Nicolás de Hidalgo.
Edificio B5; Ciudad Universitaria. CP 58030 Morelia, Michoacán, México.
C. Velázquez-Becerra / cvelazquez@ umich.mx
Facultad de Ingeniería en Tecnología de la Madera,
Laboratorio de Microbiología de la Madera,
Universidad Michoacana de San Nicolás de Hidalgo.
Edificio D, Ciudad Universitaria. CP 58030 Morelia, Michoacán, México.

RESUMEN: En importantes especies forestales se ha identificado una pérdida en la viabilidad de semilla, razón por la cual es necesario el desarrollo de estrategias que atiendan el problema, así como reconocer individuos morfológicamente superiores, favoreciendo la producción y calidad del germoplasma. El presente trabajo, evaluó la viabilidad y germinación de Cordia elaeagnoides en los municipios de Tumbiscatío y La Huacana del Estado de Michoacán, México, generando información que apoye su aprovechamiento. Fueron seleccionados ocho árboles semilleros de acuerdo a los protocolos de la "International Seed Testing Association (ISTA)", a partir de ellos, se obtuvieron muestras de semilla para realizar diversas pruebas de viabilidad y germinación. Encontrando que las semillas procedentes de la zona media/alta de la copa del árbol presentaron mayor porcentaje de viabilidad, diámetro y longitud, en comparación con las procedentes de la zona baja de la copa. Asimismo, se encontró que la temperatura afecta substancialmente la germinación, obteniendo un $53 \%$ a $30^{\circ} \mathrm{C}$. En el complejo proceso de germinación en semillas ortodoxas y recalcitrantes, factores como la temperatura y reguladores del crecimiento vegetal están fuertemente vinculados y la perturbación de su ambiente natural afecta directamente la homeostasis metabólica en semilla. La energía de germinación en $C$. elaeagnoides se ha reportado como bajo, pero puede aumentar significativamente, convirtiéndose en una opción para su producción.

Palabras clave: Manejo forestal, germoplasma, especies maderables, Cordia exsucca Sessé \& Moc.

ABSTRACT: A loss in seed viability has been identified in important forest species, which is why it is necessary to develop strategies that address the problem, as well as to recognize morphologically superior individuals to favor the production and quality of germplasm. The present work evaluated the viability and germination of Cordia elaeagnoides in the municipalities of Tumbiscatío and La Huacana in the state of Michoacán, México, generating information that supports their use. Eight seed trees were selected according to the protocols of the International Seed Testing Association 
(ISTA), and seed samples were obtained to perform various viability and germination tests, and found that the seeds coming from the middle/high zone of the tree crown had a higher percentage of viability, diameter and length, in comparison with those coming from the low zone of the crown. Likewise, it was found that temperature substantially affects the germination, $53 \%$ at $30^{\circ} \mathrm{C}$. In the complex process of germination in orthodox and recalcitrant seeds, factors such as temperature and growth plant regulators are strongly linked, and the disturbance of their natural environment directly affects the metabolic homeostasis in seed. Germination energy in C. elaeagnoides has been reported as low, but can increase significantly, becoming an option for its production.

Key words: Forest management, germplasm, timber species, Cordia exsucca Sessé \& Moc.

\section{INTRODUCCIÓN}

Un área semillera se concibe como un rodal delimitado dentro de un bosque natural o plantación forestal, en donde la selección de fenotipos superiores o de alto rendimiento en poblaciones naturales su identificación es fundamental, ya que de ellos depende la ganancia hereditaria que se alcance (CONAFOR, 2015; Vázquez, ..., \& 2016, n.d.). Dentro de esta área se encuentran los rodales semilleros (RS), agrupaciones arbóreas de igual o distinta especie donde predominan individuos fenotípicamente deseables, donde su manejo técnicamente es dirigido al sostén o aumento de producción de semilla en calidad y cantidad (Ortiz Muñoz, Hernández Acosta, Linares Márquez, Morales Romero, \& Reblledo Camacho, 2016). Los árboles semilleros pertenecientes a un RS, han demostrado ser genéticamente superiores a través de pruebas de progenie o programa de selección (Valladolid-Ontaneda, León Mejía, \& Paredes, 2017; Vázquez et al., n.d.), además, la estrategia y acción utilizada en estos sistemas forestales están dirigidas a satisfacer la demanda inmediata de semillas de calidad, al igual de integrar las necesidades económicas, culturales y sociales de los lugares en donde se desarrollen las actividades de aprovechamiento (Muñoz F. et al., 2014). En el ámbito forestal, el interés para determinar la viabilidad de semilla se ha realizado esencialmente en especies de coníferas, tomando en cuenta características dasométricas de RS, encontrado así una relación entre el fenotipo superior del árbol y mayor viabilidad en semilla (Muñoz F. et al., 2014). La baja viabilidad del germoplasma forestal en importantes especies forestales, es un problema que requiere atención, hace falta información para entender si la pérdida de viabilidad obedece a la naturaleza de la especie o factores externos que actúan en sinergia alterando la fisiología de la semilla, v.g. el tiempo e intensidad de radiación solar recibida, que a su vez modifica las condiciones óptimas de humedad (Milošević, Vujaković, \& Karagić, 2010). Los mecanismos metabólicos involucrados en el proceso de germinación pueden ser diversos, pero en general, las semillas son categorizadas como ortodoxas o recalcitrantes, las primeras se distinguen por mantener un periodo de secado durante su maduración, las cuales pueden permanecer almacenadas durante largos periodos de tiempo, por su parte, las semillas recalcitrantes no presentan estos atributos (Pammenter \& Berjak, 2014). Sin embargo, a pesar de las favorables características que las semillas ortodoxas poseen, se tiene información en donde el detrimento de viabilidad se ha notado en especies como Cordia alliodora (Ruiz \& Pav.) Oken (1833), Terminalia amazonia (Gmel.) Exell y Bursera bipinnata (Moc. \& Sessé ex DC.) Engl., esto debido a factores como un prolongado e inadecuado periodo de almacenamiento o deficiencias en las técnicas para su colecta en semilla. También, otros autores (Orantes-García, PérezFarrera, Rioja-paradela, \& Garrido-Ramírez, 2013) señalan que la viabilidad del germoplasma que ha disminuido debido a largos periodos de almacenamiento, puede aumentar parcialmente mediante tratamientos pregerminativos. Al encontrar que germoplasma recién colectado presenta más del $90 \%$ de viabilidad y puede disminuir hasta un $15 \%$ en la especie B. bipinnata, $34 \%$ en $C$. alliodora y $18 \%$ en $T$. amazonia después de 12 meses de almacenamiento. Una eficiente estrategia utilizada que ha incrementado la germinación en semilla ha sido el uso de ácido giberélico y escarificación, procedimiento con el cual, la germinación aumenta hasta $63 \%$ en B. bipinnata, C. alliodora $62 \%$ y $T$. amazonia $54 \%$. Del mismo modo, existen otras especies forestales de interés maderable que al igual presentan fuertes problemas de vialidad, tal es el 
caso de Cordia elaeagnoides A. DC., especie de amplia distribución nacional (vertiente del Pacifico, desde Jalisco hasta Chiapas), de importancia maderable por lo que muchas de sus poblaciones han sido fuertemente explotadas (Rendón-Carmona, Martínez-Yrízar, Maass, Pérez-Salicrup, \& Búrquez, 2013). En un estudio realizado sobre C. elaeagnoides, se reporta que bajo condiciones naturales presenta dificultades en su germinación, mostrando problemas relacionados con el aborto de embriones, además de ser atacadas por roedores generalistas, limitando su reproducción y propagación (Santacruz, Castañeda, Gaspar, Núñez, \& Mora, 2014), presenta también semilla con baja viabilidad, que podrían deberse a factores como la alternancia de temperatura debida a la variabilidad climática o deficiencia en factores ecológicos como polinización, el avance de tierras agrícolas y ganaderas, así como la pérdida y fragmentación de su ecosistema. Además, C. elaeagnoides exhibe una fuerte y alarmante problemática ecológica, ya que su ecosistema se encuentra bajo amenaza, constantemente es talada de forma ilegal, a pesar de ser reconocida entre las 10 especies prioritarias para la reforestación y programas de plantaciones forestales comerciales (CONAFOR, 2015). En la zona de tierra caliente en Michoacán, los pobladores han situado esta especie entre las más importantes, sustentando su importancia en el fortalecimiento de la industria artesanal regional para muebles y objetos de arte popular, debido a su alta durabilidad, resistencia, usos medicinales y ornamentales (Oza \& Kulkarni, 2017), específicamente en los municipios de Tumbiscatío y La Huacana, Cordia elaeagnoides ha sido duramente explotada, principalmente para obtención de leña, cambio de uso de suelo debido al avance de tierras agrícolas y ganaderas. Asimismo, es importante mencionar que en la región que comprenden los municipios mencionados, se tiene particular interés de utilizar especies nativas en actividades de reforestación. De acuerdo con el Programa de Manejo Reserva de la Biósfera ZicuiránInfiernillo (SEMARNAT \& CONANP, 2014), de los cuatro municipios como "Churumuco y Tumbiscatío" son los menos explorados, mientras que la zona de la sierra "Las Cruces" y faldas del cerro "Condémbaro" en el municipio de La Huacana, son hasta el momento la porción mejor conocida desde el punto de vista florístico. Sin embargo, se trata de una de las regiones más ricas en especies de plantas en Michoacán.

A pesar de existentes estrategias empleadas en mejorar la viabilidad en semilla, estas aun no han sido probabas en semillas de $C$. elaeagnoides, además, el establecimiento metodológico no está contemplado para condiciones de campo. Por esta razón, debido a que $C$. elaeagnoides es un árbol de importancia maderable, ecológica y social, reconocida como prioritaria para la reforestación y programas de plantaciones forestales comerciales, el objetivo del presente estudio fue conocer la viabilidad y germinación de esta especie arbórea, aportando conocimiento en el desarrollo de una estrategia metodológica que promueva su aprovechamiento en campo.

\section{MATERIAL Y MÉTODOS}

\section{Ubicación del área de estudio y diagnóstico de rodales semilleros de C. elaeagnoides}

El material biológico utilizado se obtuvo de los municipios de Tumbiscatío (Longitud: 102.376667, Latitud: 18.524722) y La Huacana (Longitud: -101.806389, Latitud: 18.962222) del Estado de Michoacán, México. La descripción de los predios con RS se realizó con la colaboración de la Comisión Nacional Forestal, Gerencia Estatal Michoacán, donde se obtuvo el listado de los estudios técnicos para el aprovechamiento forestal no maderable y recolección de germoplasma forestal de $C$. elaeagnoides, realizados en base a los criterios de Manual de Unidades Productoras de Germoplasma Forestal (UPGF) de la Comisión Nacional Forestal (2016). Posteriormente se realizó el diagnóstico obteniendo seis inventarios con $C$. elaeagnoides, las poblaciones naturales seleccionadas se localizaron en el área geográfica de distribución ubicada a altitudes entre los 297 y 362 metros sobre el nivel del mar, de los cuales se tomaron muestras en los sitios Manila (I), Manila (II), Caja (III) y Cuirindales (IV) (cuadro 1 y fig. 1). 
Cuadro 1. Ubicación y características dasométricas de los árboles semilleros, BS1(h')w (Semiárido cálido).

\begin{tabular}{ccccccc}
\hline $\begin{array}{c}\text { Árbol Semillero } \\
\text { (Localidad) }\end{array}$ & $\begin{array}{c}\text { Diámetro } \\
(\mathbf{m})(\mathbf{D A P})\end{array}$ & $\begin{array}{c}\text { Altura } \\
\text { del árbol } \\
(\mathbf{m})\end{array}$ & $\begin{array}{c}\text { Diámetro } \\
\text { de la copa } \\
(\mathbf{m})\end{array}$ & $\begin{array}{c}\text { Exposición } \\
\text { solar } \\
\text { (grados) }\end{array}$ & $\begin{array}{c}\text { Latitud } \\
\text { Norte }\end{array}$ & $\begin{array}{c}\text { Longitud } \\
\text { Oeste }\end{array}$ \\
\hline 1 (Manila I) & 0.46 & 12 & 7 & 31 & $18^{\circ} 45^{\prime} 59.08^{\prime \prime}$ & $102^{\circ} 16^{\prime} 53.10^{\prime \prime}$ \\
2 (Manila I) & 0.58 & 9 & 6 & & $18^{\circ} 45^{\prime} 53^{\prime} .37^{\prime \prime}$ & $102^{\circ} 17^{\prime} 0.73^{\prime \prime}$ \\
3 (Manila II) & 0.57 & 10 & 7 & 31 & $18^{\circ} 45^{\prime} 54.18^{\prime \prime}$ & $102^{\circ} 16^{\prime} 52.94^{\prime \prime}$ \\
4 (Manila II) & 0.41 & 9 & 5 & & $18^{\circ} 45^{\prime} 55.50^{\prime \prime}$ & $102^{\circ} 16^{\prime} 50.47^{\prime \prime}$ \\
5 (Caja III) & 0.48 & 10 & 8 & 218.3 & $18^{\circ} 49^{\prime} 46.27^{\prime \prime}$ & $101^{\circ} 53^{\prime} 36.57^{\prime \prime}$ \\
6 (Caja III) & 0.63 & 10 & 8 & & $18^{\circ} 49^{\prime} 46.27^{\prime \prime}$ & $101^{\circ} 53^{\prime} 35.28^{\prime \prime}$ \\
7 (Cuirindales IV) & 0.71 & 9 & 7 & 69.7 & $18^{\circ} 44^{\prime} 56.36^{\prime \prime}$ & $102^{\circ} 17^{\prime} 06.45^{\prime \prime}$ \\
& & & & & & \\
8 (Cuirindales IV) & 0.59 & 9 & 6 & & $18^{\circ} 44^{\prime} 56.75^{\prime \prime}$ & $102^{\circ} 17^{\prime} 77.53^{\prime \prime}$ \\
\hline Promedio & 0.55 & 9.75 & 6.75 & & &
\end{tabular}

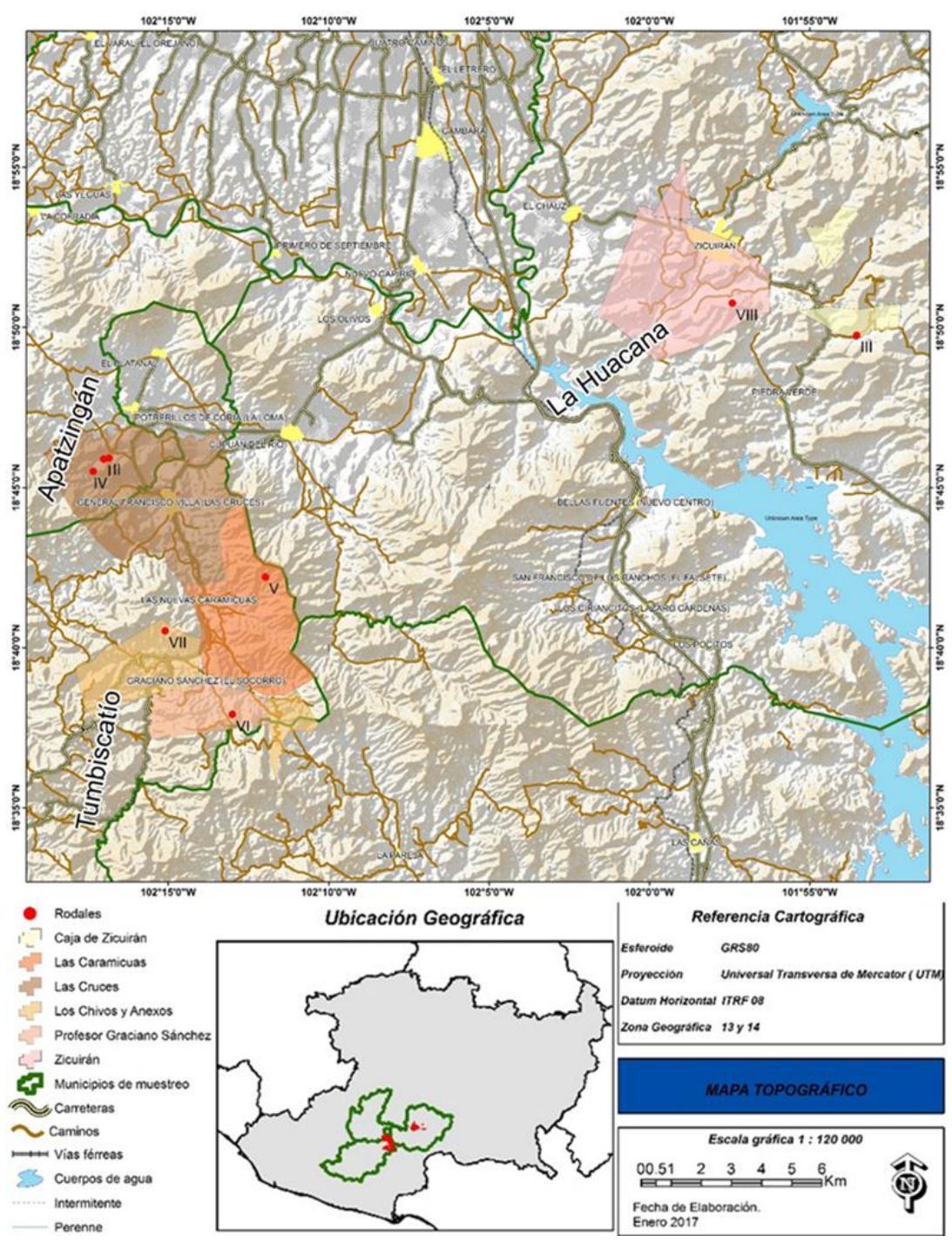

Fig 1. Rodales semilleros dirigidos para el aprovechamiento forestal no maderable y recolección de germoplasma forestal de C. elaeagnoides, conforme a la información obtenida por la Comisión Nacional Forestal (2016). Manila (I), Manila (II), Caja (III) y Cuirindales (IV). 


\section{Selección de árboles semilleros de C. elaeagnoides}

Con base en recientes trabajos realizados (Valladolid-Ontaneda et al., 2017) se tomaron en cuenta las características fenotípicas, se eligieron ocho árboles maduros a una distancia mínima de 100 metros entre ellos, libres de plaga o daño mecánico, con altura mínima de 9 metros, fuste limpio y recto, (copa de 5 metros de diámetro como mínimo), en etapa de fructificación con flores que mantuvieran un color café, determinado así la idoneidad de los individuos como árboles semilleros. De los árboles encontrados en los sitios de muestreo ya mencionados, se utilizaron para la colecta de germoplasma.

\section{Colecta de germoplasma}

De los ocho árboles que cumplieron con los criterios de selección establecidos, fueron identificados y georreferenciados (Guía para la Manipulación de Semillas Forestales de la FAO, 1991). De estos ejemplares arbóreos se tomaron muestras o lotes (2400 semillas aproximadamente) del germoplasma durante el mes de febrero (2016), la estrategia metodología consistió en: Primero se realizó una división en tres partes similares de acuerdo con su altura (Alta, Media y Baja), para posteriormente y con la ayuda de una lona $\left(10 \mathrm{~m}^{2}\right)$, ganchos telescópicos y escaleras, las semillas fueron colectadas por separado conforme con la división establecida. La semilla colectada se colocó en bolsas de plástico para ser trasladada al área en manejo y conservación de recursos forestales maderables-UMSNH, donde se almacenó a humedad y temperatura controlada $\left(10 \% / 20^{\circ} \mathrm{C}\right.$ respectivamente) hasta su uso (Guía para la manipulación de semillas forestales ortodoxas de la FAO). Después, utilizando los protocolos de la "International Seed Testing Association-ISTA" (Issue, 2004), de cada una de las muestras de semilla se obtuvieron datos de las principales características físicas, como su peso, longitud, diámetro, semillas por gramo, contenido de humedad y pureza (utilizando una balanza analítica y vernier digital $\left( \pm 0.001 \mathrm{~mm}\right.$; $\left.\operatorname{Truper}^{\mathrm{R}}\right)$ y rendimiento promedio de los árboles semilleros (numero de semillas por kilogramo, ver cuadro 2).

Cuadro 2. Características de las semillas de acuerdo a su rendimiento. Los resultados se examinaron con un Análisis de Varianza (ANOVA) y una posterior prueba de Tukey $(\mathrm{p}<0.05$ ). Las letras iguales denotan significancia estadística entre los estratos de la copa arbórea.

\begin{tabular}{ccccc}
\hline $\begin{array}{c}\text { Copa } \\
\text { Arbórea }\end{array}$ & $\mathbf{P M ~}(\mathbf{g})^{*}$ & $\mathbf{N S} / \mathbf{g}$ & $\begin{array}{c}\text { Rendimiento** } \\
(\text { Semillas/Kg) }\end{array}$ & $\mathbf{C H}(\boldsymbol{\%})$ \\
\hline Alta & $83.8( \pm 2.75) \mathrm{a}$ & $35.7( \pm 0.59) \mathrm{a}$ & $35,750( \pm 592.93) \mathrm{a}$ & $8.5( \pm 0.16) \mathrm{a}$ \\
Media & $78.7( \pm 1.93) \mathrm{ab}$ & $35.7( \pm 0.67) \mathrm{a}$ & $35,500( \pm 517.55) \mathrm{a}$ & $8.5( \pm 0.21) \mathrm{a}$ \\
Baja & $53.3( \pm 5.32) \mathrm{c}$ & $40.0( \pm 2.13) \mathrm{a}$ & $42,250( \pm 205.95) \mathrm{a}$ & $8.1( \pm 0.18) \mathrm{a}$ \\
\hline
\end{tabular}

Se muestra la media de los valores, $\mathrm{PM}=$ peso obtenido por muestra, $\mathrm{NS}=$ número de semillas, $\mathrm{CH}=$ contenido de humedad en semilla. *Datos basados en muestras libre de impurezas, **estimación del rendimiento basado en el número de semillas por gramo de muestra obtenida, \pm corresponde a la desviación estándar.

\section{Pruebas de viabilidad en semilla}

Tomando como referencia el trabajo de (Marcos Filho, 2015) y los protocolos de la ISTA, se realizaron cuatro pruebas para definir la viabilidad de semilla por estrato (Alto, medio y bajo), en donde se tomaron al azar 100 semillas por estrato y árbol, la estrategia metodológica consistió en:

a) Separación por flotación. Las muestras de cada estrato por árbol (100 semillas), se colocaron en un vaso de precipitados $(500 \mathrm{ml})$ y agregaron $400 \mathrm{ml}$ de agua destilada, se dejaron reposar por 12 horas a temperatura ambiente $\left(25^{\circ} \mathrm{C}\right)$. Después, se realizó un conteo de las semillas precipitadas y flotadas, los datos se reportaron en porcentaje.

b) Prueba de aspiración con aire: Utilizando un equipo separador con aire SeedTechSystems ${ }^{\mathrm{TM}}$ STS-MC3, se tomaron $100 \mathrm{~g}$ de cada muestra por estrato para este ensayo, 
a un flujo de aire de $30.5 \mathrm{~m}^{3} / \mathrm{min}$ se hicieron pasar las muestras de semilla. El principio consiste en separar las semillas de menor peso (no viables) hacia un recipiente, por su parte, las de mayor peso (viables) quedan en la parte inferior del equipo, contabilizando el porcentaje de semillas viables y no viables.

c) Tinción con tetrazolio: Muestras representativas de cada estrato se colocaron en agua destilada por 12 horas (facilitando la difusión del tetrazolio en el tejido interior), posteriormente en las semillas se realizó un corte transversal por encima del embrión. Enseguida se colocaron en la solución tetrazolio al 1\% (2,3,5 cloruro de trifenil tetrazolio, Sigma-Aldrich $\left.{ }^{\mathrm{R}}\right)$, a temperatura de $30^{\circ} \mathrm{C} / 1 \mathrm{~h}$ y condiciones de oscuridad. Después se observaron los embriones utilizando un microscopio estereoscópico (Zeiss Stemi 305EDU) a 40X, para identificar las partes teñidas del embrión (en rojo) y clasificar la viabilidad de la semilla mediante la tinción del tejido embrionario (Oliveira, Torres, Nogueira, Magno, \& Freitas, 2016).

d) Disección de semilla: Se realizó un corte transversal sobre la superficie de la testa, permitiendo observar color, textura y consistencia del endospermo, identificando el desarrollo del embrión. En este ensayo se contabilizó el número de semillas con embriones sanos y número de compartimentos o sacos embrionarios en cada muestra de las semillas viables y no viables.

\section{Germinación de semillas de Cordia elaeagnoides}

En este ensayo se utilizó una muestra de semilla tomada al azar y procedente de los ocho árboles identificados como superiores, para el cual se utilizó una cantidad de 100 semillas para cada condición (temperatura), las características del experimento consistieron en: Las semillas fueron colocadas en charolas de plástico en contenedores individuales con un volumen de 1.5 $\mathrm{cm}^{3}$ de sustrato por semilla (Agrolita:Arena:Peat-Moss en proporción 1:1:1) e hidratadas a capacidad de campo del sustrato $(2.7 \mathrm{ml}$ de agua cada 4 días aproximadamente). Las condiciones de germinación se realizaron en una cámara de crecimiento vegetal, operada bajo un entorno controlado de 16 horas luz y 8 en oscuridad y condiciones de temperatura de 25,28 y $30^{\circ} \mathrm{C}$. Las evaluaciones de germinación se realizaron a los 7, 14, 21 y 28 días después de la siembra. Finalmente, la germinación se reportó de acuerdo al trabajo realizado por (Barraza A., Benavides B., \& Torres M., 2016; Orantes-García et al., 2013), en donde se considera el numero de semillas utilizadas entre el numero de semillas germinadas por cien.

\section{Análisis estadístico}

Utilizando el Programa STATISTICA Versión 7, los resultados se examinaron con un Análisis de Varianza (ANOVA) y una posterior prueba de Tukey $(p<0.05)$.

\section{RESUltados}

Las características geográficas identificadas en los sitios de muestreo de los árboles semilleros mostraron similitud entre ellas, es decir, a pesar de la distancia entre las localidades de muestreo corresponde a kilómetros (fig. 1), las condiciones de clima (BS1(h')w), precipitación (647 a 746 $\mathrm{mm}$ ) y altitud (297 a $362 \mathrm{msnm}$ ) son cercanos y únicamente se encontraron diferencias en los valores de exposición a la radiación solar por sitio (cuadro 1).

\section{Copa arbórea y características de la semilla en Cordia elaeagnoides}

Los resultados también revelaron una diferencia en las muestras obtenidas, respectivas con el estrato de la copa arbórea, es decir, en la zona media/alta de la copa fue mayor la muestra de semilla obtenida. En el cuadro 2 se muestran los valores de peso obtenido por muestra (g), en donde, los datos mas altos corresponden a la parte media/alta del dosel, que las obtenidas en el estrato inferior o bajo. Sin embargo, al realizar el conteo de número de semillas por gramo entre estratos, no se encontró una diferencia estadísticamente significativa, situación similar que se tiene con los valores de rendimiento (cuadro 2). 
Por su parte, los datos obtenidos en diámetro y longitud de la semilla, señalan una tendencia a ser de mayor dimensión en el estrato alto, y dichos valores van disminuyendo acorde a la altura de la copa, teniendo los datos más bajos en la parte inferior del dosel (fig. 2).

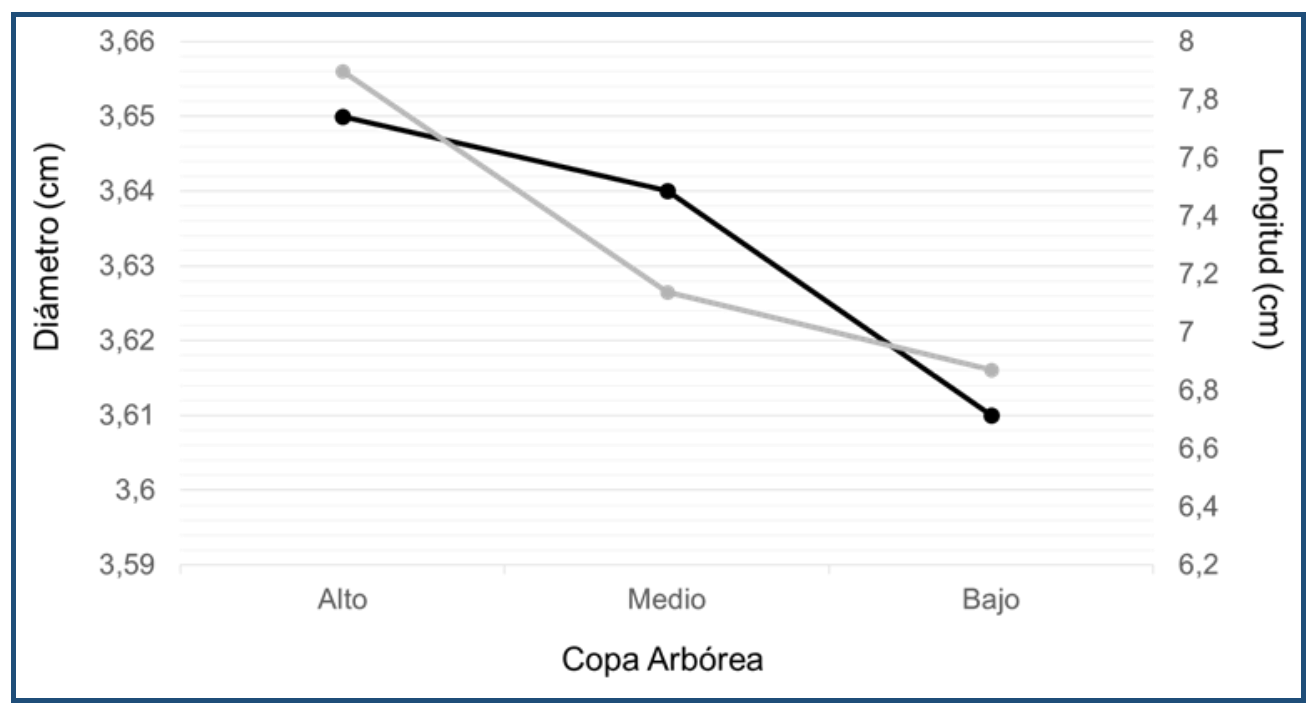

Fig 2. Diámetro y longitud de la semilla en C. elaeagnoides de acuerdo al estrato de la copa arbórea. La línea gris indica el diámetro y la línea negra indica la longitud de semilla.

\section{Viabilidad de semilla en Cordia elaeagnoides}

Los ensayos realizados para conocer la viabilidad de semilla revelaron, en la técnica de separación con aire (columna densimétrica) correspondió con una mayor viabilidad en Cordia, en relación a las complementarias pruebas, obteniendo valores de 85,77 y $71 \%$ para los estratos alto, medio y bajo respectivamente. Además, exponiendo también que la prueba con tinción de tetrazolio (para estratos medio y bajo) se obtuvieron valores similares estadísticamente a la prueba anteriormente reseñada (fig. 3). Por su parte, en el ensayo de flotación de semilla (Inmersión) se obtuvieron los valores mas bajos en viabilidad, siendo estadísticamente diferentes a las anteriores dos pruebas realizadas $(61.6,63.2$ y $59.6 \%$ para los estratos de alto a bajo respectivamente).

La principal característica fisiológica de las semillas ortodoxas es su gran tolerancia a la deshidratación, en el presente documento se evidencia que las muestras de semilla presentan contenidos de humedad de 8.5 a $8.1 \%$ (cuadro 2). De igual forma, los resultados obtenidos en el escrutinio del número de compartimentos embrionarios en semilla de Cordia, revelaron que en semillas identificadas como viables existe una relación entre el número de embriones en semilla y compartimentos embrionarios encontrados (fig. 4A), a diferencia de semillas clasificadas como no viables, que mostraron tener baja presencia de número de embriones en razón con el número de compartimentos (fig. 4B). En la figura 4C se exponen los compartimentos embrionarios en semillas de $C$. elaeagnoides, resaltando la presencia de 3 y 4 espacios principalmente. 


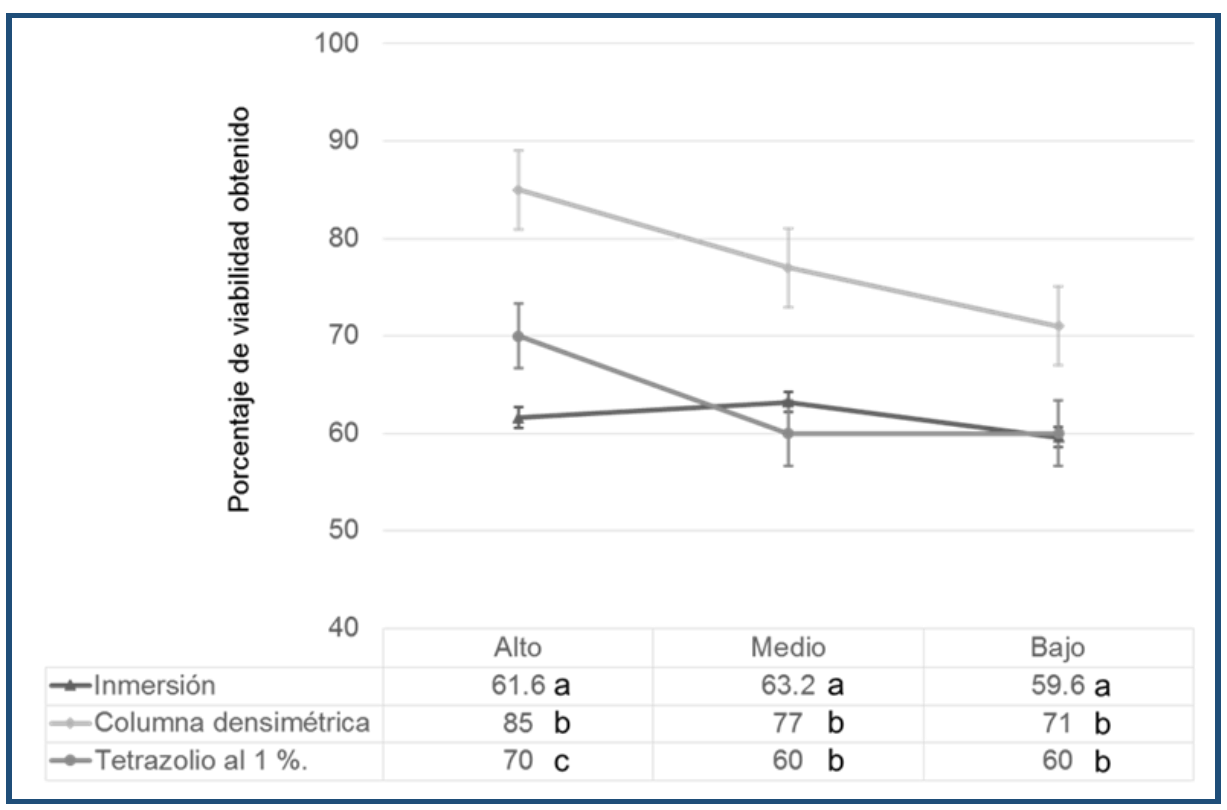

Fig 3. Porcentaje de viabilidad de semilla en C. elaeagnoides de acuerdo al estrato de la copa arbórea, los valores corresponden a la media obtenida y las barras muestran error estándar. Resultados examinados mediante un Análisis de Varianza (ANOVA) y una posterior prueba de Tukey $(\mathrm{p}<0.05$ ). Las letras iguales denotan significancia estadística entre los estratos de la copa arbórea.

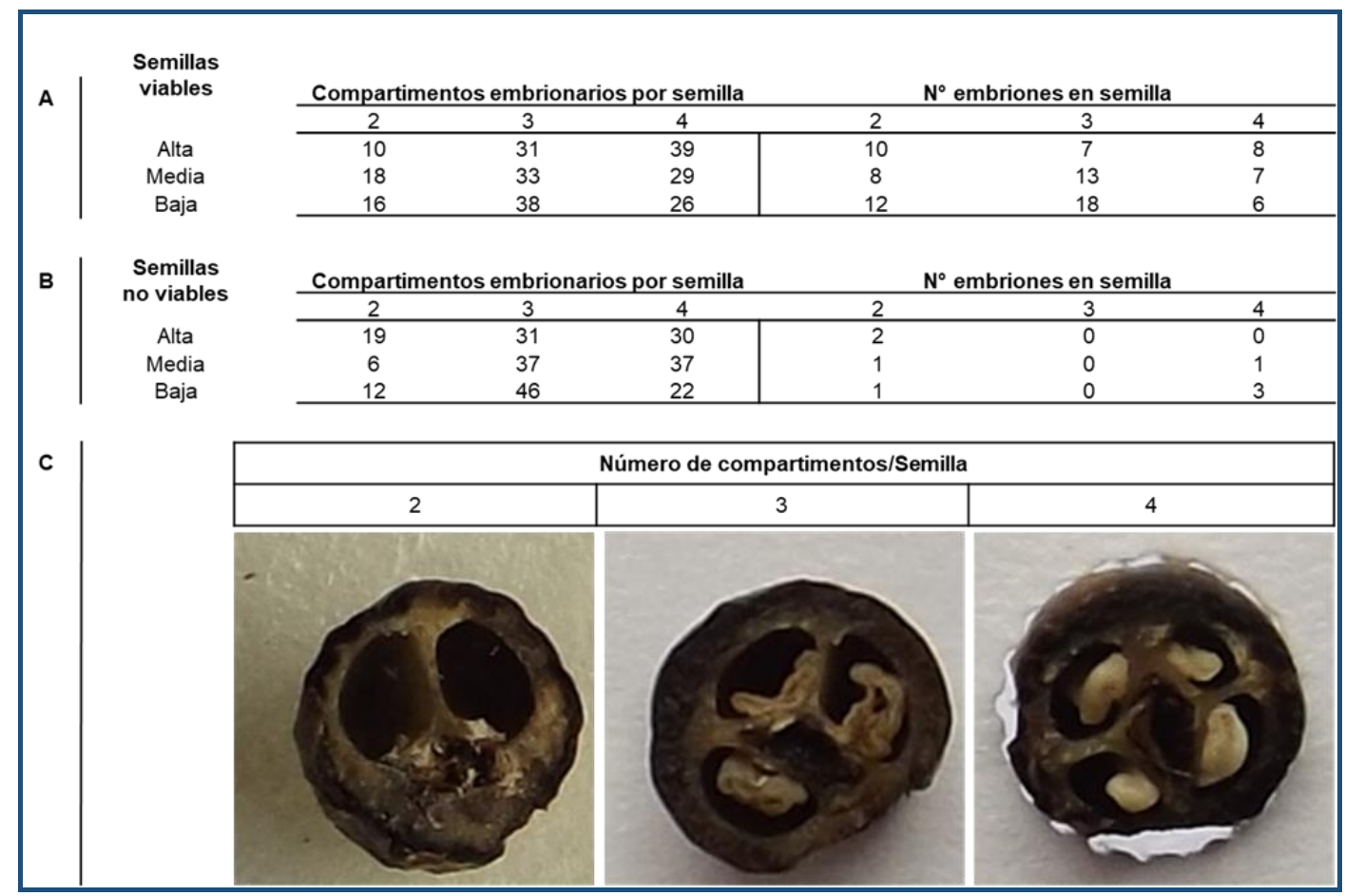

Fig 4. Compartimentos embrionarios en semilla de C. elaeagnoides de acuerdo al estrato de la copa arbórea en semillas viables y vanas. A) Semillas viables y número de embriones en semilla por compartimento embrionario, B) Semillas no viables y número de embriones en semilla por compartimento embrionario, C) Compartimentos embrionarios en semillas de C. elaeagnoides, mostrando la presencia de 2, 3 y 4 espacios. 


\section{Evaluación de la germinación en C. elaeagnoides}

La investigación realizada en este trabajo, mostró que la temperatura es una importante condición que puede limitar significativamente la germinación en $C$. elaeagnoides. En la figura 5 se muestra que bajo condiciones de $30^{\circ} \mathrm{C}$ se alcanzó una germinación acumulada de $53 \%$, disminuyendo de acuerdo con la baja de temperatura, es decir, a 28 y $25^{\circ} \mathrm{C}$ después de 28 días los valores de germinación obtenidos fueron de 0 y $42 \%$. Es importante también resaltar que, a 14 días de siembra se obtuvo el cien porciento del total final de semillas germinadas ensayadas a $30^{\circ} \mathrm{C}$, no así el experimento realizado a $28^{\circ} \mathrm{C}$, alcanzando su cien porciento del total final hasta los 21 días.

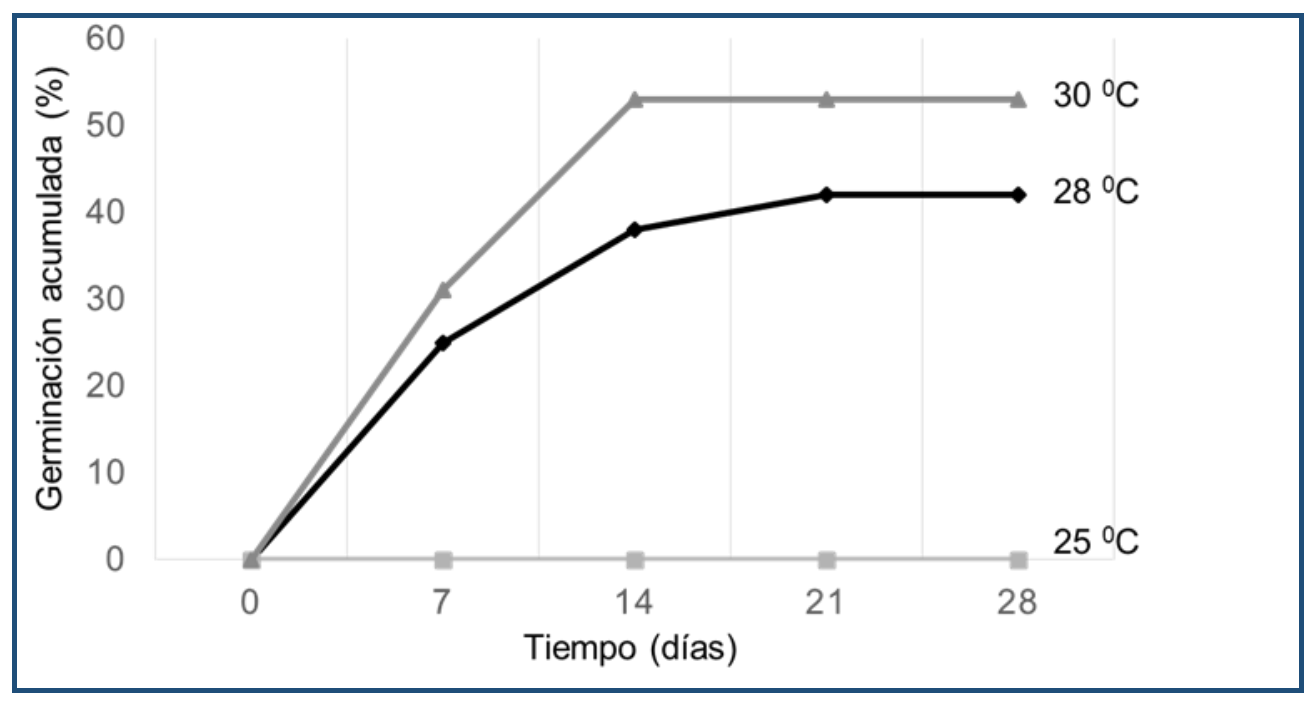

Fig 5. Germinación acumulada, en donde se muestra que a una temperatura de $30^{\circ} \mathrm{C}$ se alcanzó un $53 \%$ de semillas germinadas, después de 14 días de siembra.

\section{DISCUSIÓN}

\section{Condiciones del área de estudio}

En el proceso fisiológico de génesis y maduración de semilla, la intensidad lumínica juega un papel determinante, ya que, la luz natural puede mimetizar los efectos dados por alternancia de temperatura, efecto vinculado con la germinación (Li, Yin, Jongsma, \& Wang, 2011). En este trabajo encontramos que las condiciones climáticas entre localidades (Tumbiscatío y La Huacana Michoacán, México) son homogéneas entre ellas (BS1(h')w) y dicha cualidad es importante, ya que desde un punto de vista de producción de planta, se podrá colectar semilla de cualquiera de estos sitios, ofreciendo la certeza para poder alcanzar resultados uniformes, así como establecer las condiciones para su muestreo.

\section{Desarrollo de la semilla en C. elaeagnoides}

El desarrollo y maduración del germoplasma en los árboles no necesariamente se lleva a cabo homogéneamente en la planta, es decir, se efectúa por etapas o secciones que favorecen su continuidad en el ecosistema. Existen factores como la disponibilidad de nutrientes, temperatura, presencia de plagas, enfermedades, disposición genética o humedad, que intervienen en la maduración de semillas. En particular, elevados porcentajes de humedad durante el desarrollo de la semilla podrian mejorar la viabilidad y capacidad de almacenamiento (Gantait, Kundu, Wani, \& Das, 2016; Pammenter \& Berjak, 2014). v.g. en algunas especies de pinos (Pinus halepensis Mill), la maduración del cono está influenciada por la densidad arbórea en los RS, generando una menor competencia entre los pinos y la flora del bosque que los 
acompaña, lo que resulta en un aumento dimensional general de los árboles, incluida la producción de semillas. Sugiriendo que la mejora de las particularidades morfológicas del árbol podría favorecer las características de semillas y cultivo de conos, es decir, la producción de semillas y conos es mayor en árboles que presentan un dosel superior en altura y diámetro (Ayari \& Khouja, 2014). A largo plazo, la calidad de las semillas se puede ver reflejada en los rendimientos y desarrollo de los árboles en campo, disminuyendo los costos de producción y manejo.

Información publicada por CONAFOR, reporta que las semillas de Cordia son ortodoxas, que además presentan valores de viabilidad estimados bajo condiciones de almacenamiento del $50 \%$, sin embargo, en este trabajo se alcanzó valores de viabilidad del hasta $85 \%$. Aunque el término viabilidad y germinación pudieran relacionarse, en el presente trabajo se identificaron valores de viabilidad (dependiendo de la prueba utilizada) entre 59.6 a $85 \%$, los cuales no correspondieron a los valores de germinación encontrados (53\%), lo cual nos dice que en $C$. elaeagnoides no necesariamente semillas con elevados valores de viabilidad se verán reflejados en la germinación, ya que, para una germinación exitosa en condiciones in vitro, vivero o campo es necesario encontrar las condiciones ambientales ideales.

La CONAFOR también ha reportado que estas semillas pueden almacenarse a temperatura de hasta $0^{\circ} \mathrm{C}$ y mantener su viabilidad por varios años, sin embargo, en este trabajo también se encontró que la viabilidad se pierde con el tiempo a una taza inusual para semillas ortodoxas (Walters, 2015), ya que las mismas pruebas realizadas seis meses después, se obtuvo una pérdida en la viabilidad del 50\% aproximadamente (datos no mostrados). En la actualidad, en esta especie o género taxonómico no se ha reportado una relación con el proceso de maduración y viabilidad de semilla. Pero se conoce que, durante el ciclo de desarrollo de las semillas ortodoxas, estas desarrollan gran parte de las características de tolerancias al estrés hídrico, y durante este proceso es importante que la condición ambiental al cual el árbol esta sometido no se vean alteradas o pasen por fuertes cambios. En etapas iniciales de desarrollo y crecimiento del embrión (formación de semillas), cuando la actividad metabólica y contenido de agua es alto, las semillas son sensibles a la desecación, por su lado, en la fase de maduración, las semillas ortodoxas son inducidas a tolerar la desecación, hasta el comienzo de la germinación (Pereira et al., 2017). También, es importante tener en cuenta que una mejor manera de clasificar si una semilla es ortodoxa seria considerando su potencial hídrico, ya que aun existen dudas en categorizar si una semilla es recalcitrante, intermedia u ortodoxa en base a su capacidad a la desecación.

\section{Germinación en C. elaeagnoides}

Se encontró que bajo condiciones de $30^{\circ} \mathrm{C}$ se obtuvo el porcentaje de germinación mas alto, que coindice con la temperatura media alcanzada en la región de "Tierra Caliente" Michoacán $\left(27.8^{\circ} \mathrm{C}\right)$, por lo cual, significaría que el productor podrá realizar el procedimiento de germinación de semilla sin la necesidad de adquirir equipo costoso o que represente dificultades en operación y/o mantenimiento. El papel que desempeña la temperatura en semilla se extiende desde su génesis, pasando por el almacenamiento y germinación, por ejemplo, en un trabajo publicado y utilizando como modelo de estudio semillas de Ginkgo biloba L. se encontró que el comportamiento de la semilla durante un almacenamiento a $25^{\circ} \mathrm{C}$, después de seis meses pierden el total de su viabilidad. En cambio, al ser almacenadas a una baja temperatura $\left(4^{\circ} \mathrm{C}\right) \mathrm{se}$ conserva la viabilidad hasta 12 meses, no así su capacidad de germinación, ya que disminuyó considerablemente después de seis meses. Los autores del trabajo cometan que en tejido embrionario la presencia de dos importantes antioxidantes como el ascorbato y glutatión, muestran un comportamiento diferente en las dos condiciones de temperatura ensayadas, regulando el estrés oxidativo durante el almacenaje, manifestándose en la germinación (Tommasi, Paciolla, Concetta de Pinto, \& De Gara, 2006). Y como anteriormente se a visto, aunque una semilla pueda tener un tejido embrionario morfológicamente completo y desarrollado, no necesariamente la germinación se llevaría a cabo, pero si es un importante indicador de viabilidad. En otro estudio, utilizando como modelo semillas de Jatropha Curcas 
Linn., se analizó el efecto de la temperatura y medio de crecimiento sobre la germinación, encontrando que del rango de temperatura ensayada $\left(20-37^{\circ} \mathrm{C}\right)$, a $30^{\circ} \mathrm{C}$ se registró el mayor porcentaje de germinación (84\%), notando una tendencia relacionada a la temperatura, demostrando la influencia de este factor (Gairola, Nautiyal, \& Dwivedi, 2011).

También, un estudio sobre la viabilidad a diferente temperatura durante un período de almacenamiento de cinco años sobre semillas de Brassica repanda, Eruca vesicaria, Malcolmia littorea, Moricandia arvensis, Rorippa nasturtium-aquaticum, Sinapis alba, Sisymbrium runcinatum, almacenadas en 48 distintos ambientes que comprendían una combinación de ocho contenidos de agua (0.21 a 0.01 g.H2H.g-1 DW) y seis distintas condiciones de temperatura durante su almacenaje $\left(45,35,20,5,-25,-170{ }^{\circ} \mathrm{C}\right)$. Se encontró que el contenido de agua es crítico durante el almacenamiento de semillas a temperatura elevada, el efecto de la desecación extrema a $45^{\circ} \mathrm{C}$ mostró efectos dañinos manifestados en inhibir la germinación (Mira, Estrelles, \& González-Benito, 2015).

Finalmente, en otro manuscrito se reporta que, utilizando cuatro especies arbóreas (dos especies de Fabaceae y dos Bignoniaceae) se encontró una respuesta a la temperatura $\left(25 / 30^{\circ} \mathrm{C}\right) \mathrm{y}$ determinante para promover la germinación de la mayoría de las semillas ensayadas. El germoplasma de las cuatro especies presentó un comportamiento propio de semillas ortodoxas, mostrando tolerancia y germinación en condiciones ambientales con intervalos amplios de temperatura y luminosidad (Vargas-Figueroa, 2015). Pese a que la temperatura desempeña un papel importante en el fenómeno de germinación, con la evidencia mostrada con anterioridad resulta evidente que el proceso de germinación es multifactorial, v.g. La inducción de la dormancia en semilla ocurre durante la maduración y está regulada por el desarrollo. Los factores ambientales experimentados por la planta progenitora durante la maduración de la semilla, como temperatura, luz y nitrato del suelo, influyen en el nivel de dormancia. Esto influye de manera que, cuando las condiciones ambientales favorecen la siguiente temporada de crecimiento, este mecanismo puede ayudar a la próxima generación (memoria celular) a adaptarse al entorno. Especialmente, la temperatura ha demostrado ser un factor determinante, ya que, utilizando la planta Arabidopsis sp., se vio que bajas temperaturas durante la maduración de las semillas aumentan los niveles de dormancia. Evolutivamente esto ha permitido evitar que todas las semillas germinen simultáneamente, reduciendo el riesgo de mortalidad en la progenie (Née, Xiang, \& Soppe, 2017). Del mismo modo, los reguladores del crecimiento vegetal como las giberelinas (AG), están fuertemente implicadas en los procesos de germinación, y se sabe que existen genes que regulan la expresión de AG, dependientes de la temperatura, capaces de alterar el metabolismo de la semilla. Estos efectos conducen a un debilitamiento del endospermo que determinan el tiempo óptimo para germinar (Graeber et al., 2014).

\section{Conclusiones}

A pesar de existir una importante presencia de árboles de C. elaeagnoides en los sitios de estudio (Tumbiscatío y La Huacana, Michoacán), únicamente un bajo porcentaje cuentan con las características necesarias para ser categorizados como árboles superiores. Además, de los ocho árboles superiores del cual se realizó la recolección de germoplasma, la parte media/alta de la copa del árbol presentó mejor rendimiento, semillas de mayor talla y mayor porcentaje de viabilidad. También se encontró que, el efecto de la temperatura sobre la germinación en semillas de $C$. elaeagnoides fue determinante para alcanzar un 53\%, ya que, al disminuir la temperatura del bioensayo, también disminuye la germinación. 


\section{AGRAdecimientos}

Al CONACYT por el apoyo financiero a este trabajo, a través del proyecto número 263424 , y al CIC-UMSNH por su soporte institucional.

\section{LITERATURA CITADA}

Ayari, A., \& Khouja, M. L. (2014). Ecophysiological variables influencing Aleppo pine seed and cone production: A review. Tree Physiology, 34(4), 426-437. https://doi.org/10.1093/treephys/tpu022

Barraza A., F., Benavides B., O., \& Torres M., F. (2016). Calidad fisiológica y energía de germinación de semillas de balsamina (Momordica charantia L.). Revista de Ciencias Agrícolas, 33(1), 43. https://doi.org/10.22267/rcia.163301.5

CONAFOR. (2015). Programa de Desarrollo de Plantaciones Forestales Comerciales A 15 años de su creación. 1-198.

Gairola, K. C., Nautiyal, A. R., \& Dwivedi, A. K. (2011). Effect of temperatures and germination media on seed germination of Jatropha curcas Linn. Advances in Bioresearch, 2(2), 66-71.

Gantait, S., Kundu, S., Wani, S. H., \& Das, P. K. (2016). Cryopreservation of Forest Tree Seeds: A Mini-Review. Journal of Forest and Environmental Science, 32(3), 311-322. https://doi.org/10.7747/JFES.2016.32.3.311

Graeber, K., Linkies, A., Steinbrecher, T., Mummenhoff, K., Tarkowska, D., Ture kova, V., ... Leubner-Metzger, G. (2014). DELAY OF GERMINATION 1 mediates a conserved coat-dormancy mechanism for the temperature- and gibberellin-dependent control of seed germination. Proceedings of the National Academy of Sciences, 111(34), E3571E3580. https://doi.org/10.1073/pnas.1403851111

Issue, I. N. T. (2004). Seed Testing. World, (127), 45-48. https://doi.org/10.1081/E-EPCS

Li, J., Yin, L. Y., Jongsma, M. A., \& Wang, C. Y. (2011). Effects of light, hydropriming and abiotic stress on seed germination, and shoot and root growth of pyrethrum (Tanacetum cinerariifolium). Industrial Crops and Products, 34(3), 1543-1549. https://doi.org/10.1016/j.indcrop.2011.05.012

Marcos Filho, J. (2015). Seed vigor testing: an overview of the past, present and future perspective. Scientia Agricola, 72(4), 363-374. https://doi.org/10.1590/0103-90162015-0007

Milošević, M., Vujaković, M., \& Karagić, D. (2010). Vigour tests as indicators of seed viability. Genetika, 42(1), 103-118. https://doi.org/10.2298/GENSR1001103M

Mira, S., Estrelles, E., \& González-Benito, M. E. (2015). Effect of water content and temperature on seed longevity of seven Brassicaceae species after 5 years of storage. Plant Biology, 17(1), 153-162. https://doi.org/10.1111/plb.12183

Muñoz F., H. J., Muñoz G., J., Hernández A., H., García M., J. J., Coria A., V. M., \& Hernández R., J. (2014). Caracterización dasométrica de tres rodales semilleros de especies del género Pinus en el estado de Guerrero, México. Foresta Veracruzana, 16(2), 23-30. https://doi.org/10.2225/vol11-issue4-fulltext-2

Née, G., Xiang, Y., \& Soppe, W. J. (2017). The release of dormancy, a wake-up call for seeds to germinate. Current Opinion in Plant Biology, 35, 8-14. https://doi.org/10.1016/j.pbi.2016.09.002

Oliveira, F. N. De, Torres, S. B., Nogueira, N. W., Magno, R., \& Freitas, O. De. (2016). Viability of Simira gardneriana M . R. Barbosa \& Peixoto seeds by the tetrazolium test 1. 7-13.

Orantes-García, C., Pérez-Farrera, M. Á., Rioja-paradela, T. M., \& Garrido-Ramírez, E. R. (2013). Viabilidad Y Germinación De Semillas De Tres Especies Arbóreas Nativas De La Selva Tropical, Chiapas, México. Polibotánica, 36, 117-127.

Ortiz Muñoz, E., Hernández Acosta, C. C., Linares Márquez, P., Morales Romero, Z., \& Reblledo Camacho, V. (2016). Selección de árboles semilleros de Juglans pyriformis 
Recibido:

18/diciembre/2018

Aceptado:

27/mayo/2019
Liebm . en poblaciones naturales de Coatepec y Coacoatzintla, Veracruz Juglans pyriformis Liebm . seed tree selection in natural populations of Coatepec and Coacoatzintla , Veracruz. Rev. Mex. Cien. For., 7(38), 43-58. Retrieved from http://www.redalyc.org/articulo.oa?id=63450027003\%0ACómo

Oza, M. J., \& Kulkarni, Y. A. (2017). Traditional uses, phytochemistry and pharmacology of the medicinal species of the genus Cordia (Boraginaceae). Journal of Pharmacy and Pharmacology, 69(7), 755-789. https://doi.org/10.1111/jphp.12715

Pammenter, N. W., \& Berjak, P. (2014). Physiology of Desiccation-Sensitive (Recalcitrant) Seeds and the Implications for Cryopreservation. International Journal of Plant Sciences, 175(1), 21-28. https://doi.org/10.1086/673302

Pereira, W. V. S., Faria, J. M. R., José, A. C., Tonetti, O. A. O., Ligterink, W., \& Hilhorst, H. W. M. (2017). Is the loss of desiccation tolerance in orthodox seeds affected by provenance? South African Journal of Botany, 112, 296-302. https://doi.org/10.1016/j.sajb.2017.06.008

Rendón-Carmona, H., Martínez-Yrízar, A., Maass, J. M., Pérez-Salicrup, D. R., \& Búrquez, A. (2013). La extracción selectiva de vara para uso hortícola en México: Implicaciones para la conservación del bosque tropical caducifolio y sus recursos. Botanical Sciences, 91(4), 493-503.

Santacruz, F., Castañeda, J., Gaspar, A., Núñez, N., \& Mora, A. (2014). Rompimiento de la dormancia en semillas y propagación in vitro de Cordia elaeagnoides A . DC . Breaking of dormancy in seeds and in vitro propagation of. Revista Mexicana de Ciencias Forestales, 5(25), 84-97. Retrieved from http://www.scielo.org.mx/scielo.php?script=sci_arttext\&pid=S2007$11322014000500007 \& \operatorname{lng}=$ es\&tlng=es.

SEMARNAT, \& CONANP. (2014). Programa de Manejo Reserva de la Biósfera ZicuiránInfiernillo. Igarss 2014, (1), 270. https://doi.org/10.1007/s13398-014-0173-7.2

Tommasi, F., Paciolla, C., Concetta de Pinto, M., \& De Gara, L. (2006). Effects of storage temperature on viability, germination and antioxidant metabolism in Ginkgo biloba L. seeds. Plant Physiology and Biochemistry, 44(5-6), 359-368. https://doi.org/10.1016/j.plaphy.2006.06.014

Valladolid-Ontaneda, J., León Mejía, Á., \& Paredes, D. (2017). Selección de árboles semilleros en plantaciones forestales de la provincia de Santa Elena, Ecuador. Revista Científica y Tecnológica UPSE, IV(2), 105-110.

Vargas-Figueroa, J. A. (2015). Germinación de semillas de cuatro especies arbóreas del bosque seco tropical del Valle del Cauca, Colombia. Revista de Biología Tropical, 63(1), 249. https://doi.org/10.15517/rbt.v63i1.14123

Vázquez, B. G., ... E. C. O.-R. mexicana, \& 2016, undefined. (n.d.). Selección de árboles sobresalientes de caoba (Swietenia macrophylla King.) en un rodal natural mediante métodos multivariados. Scielo.Org.Mx. Retrieved from http://www.scielo.org.mx/scielo.php?pid=S2007$11322016000500051 \&$ script=sci_arttext

Walters, C. (2015). Orthodoxy, recalcitrance and in-between: describing variation in seed storage characteristics using threshold responses to water loss. Planta, 242(2), 397406. https://doi.org/10.1007/s00425-015-2312-6 